\title{
The Stressors and Psychological Well-Being of Lesbian, Gay and Bisexual among Chinese Adults in Hong Kong
}

\author{
Lai Sheung Winnie Cheng \\ School of Nursing, Tung Wah College, Hong Kong, China \\ Email: winniecheng@twc.edu.hk
}

How to cite this paper: Cheng L.S.W. (2019) The Stressors and Psychological Well-Being of Lesbian, Gay and Bisexual among Chinese Adults in Hong Kong. Journal of Biosciences and Medicines, 7, $65-70$.

https://doi.org/10.4236/jbm.2019.75010

Received: April 10, 2019

Accepted: May 14, 2019

Published: May 17, 2019

\begin{abstract}
Background: Lesbian, Gay and Bisexual (LGB) are individuals who often experienced stigma and discrimination which has detrimental effects on psychological well-being. Objectives: This study aimed to identify the major stressors and psychological well-being of LGB among Chinese adults in Hong Kong. Methods: A cross-sectional study was employed using self-administered questionnaires. Convenience sampling was used to recruit subjects. Demographic information, Measure of Gay-related stress (MOGS) and The Center for Epidemiologic Studies Depression Scale (CES-D Scale) were used to collect data. Results: The major stressor among LGB individuals in Hong Kong was family reaction. Majority of them had a high level of depression. Conclusion: LGB individuals in Hong Kong experienced high level of depression.
\end{abstract}

\section{Keywords}

Lesbian, Gay and Bisexual, Depression, Sexual Minority, Minority Stress

\section{Introduction}

Stress in daily life is unavoidable but some groups of people are more likely to experience stressful life events. One such minority group-lesbian, gay, and bisexual (LGB) may be more likely to experience stress due to stigma and discrimination [1]. Sexual minorities are victimized by not only being verbally harassed but also physically assaulted [2]. Psychological distress is experienced by sexual minority individuals across different age group-secondary students [3], adolescents [4], and older adults [5]. Numerous studies suggested that they encounter stressful experience which contributes to mental health problem such as 
anxiety and depression [5] [6] [7].

LGB individuals in Chinese societies-predominant by Yin-Yang harmony of heterosexual relationship [8] —are expected to face prejudice and discrimination against them [9]. However, little is known about the psychological well-being of LGB among Chinese adults in Hong Kong.

The purpose of this study sought to examine the risk of depression in LGB among Chinese adults in Hong Kong. The focus of this paper reported the minority stressors experienced by LGB individuals and their risk of depression. In line with the literature on psychological distress among LGB, it was expected that hose with higher levels of minority stress would report higher risk of depression.

\section{Methods}

\subsection{Design}

A cross-sectional study was conducted in online form between February and March 2017. The sample was recruited through convenience sampling. Respondents were required to identify as lesbian, gay, or bisexual, be aged 18 or over, and be Chinese ethnic and primarily resident in Hong Kong. The online questionnaire was distributed by Lesbian, Gay, Bisexual and Transgender (LGBT) organization in Hong Kong through social media, such as Facebook and Whats App. The study was approved by Human Research Ethics Committee of the author's institution.

\subsection{Questionnaires}

Validated questionnaires were used to measure minority stress and levels of depression level. Minority stress was measured by four subscales of the Measure of Gay-related stress (MOGS) [10], including ("Family Reaction Scale" 9-item), "Visibility with Family/Friends Scale" (7-item), "Visibility with School/Public Scale" (6-item) and "Sexual Orientation Conflict Scale" (4-item). There were 26 items from the four subscales. Respondents were then asked to rate on a 4-point Likert scale for each item, ranging from 0 (not at all stressful) to 4 (extremely stressful). The factor loading of the four subscales ranged from 0.51 to 0.84 with variance of $63.5 \%$. The reliability coefficients indicated good internal consistency and ranged from 0.71 to 0.90 [10].

The Center for Epidemiologic Studies Depression Scale (CES-D) was used to measure the levels of depression [11]. This scale consist of 20 items to ask respondent to rate how often over the past week they experienced symptoms associated with depression, such as restless sleep, poor appetite, and feeling lonely. The scale was measured with 4-point Likert scale ranging from 0 to 3 for each item $(0=$ Rarely or None of the Time, $1=$ Some or Little of the Time, $2=$ Moderately or Much of the time, 3 = Most or Almost All the Time). Scores range from 0 to 60 , with high scores indicating greater depressive symptoms. The CES-D had been recognized and accepted for good sensitivity and specificity and 
high internal consistency [12].

Demographic information was collected on age, gender, relationship status, education level, monthly income, religion and sexual identity.

\subsection{Data Analysis}

All the data were analyzed by IBM SPSS Statistics version 23. Descriptive statistics were used to measure frequencies and percentages. Bivariate correlations were used to analyze the relationships between the stressors and levels of depression. The alpha was set at 0.05 .

\section{Results}

One hundred and seventy-five people participated in this study. Usable questionnaires were 161. The participants had a mean age of $26.1(\mathrm{SD}=7.5)$ years. They identified their sexual identity as lesbian (38.5\%), gay (30.4\%), and bisexual (31.1\%), and their gender identity as female (61.5\%), male (37.9\%), and gender fluid $(0.6 \%)$. Table 1 presents the demographic characteristics.

Table 2 presents the descriptive statistics for the minority stress subscales and depression levels and the bivariate correlations. Family reaction scale (mean = $1.75, \mathrm{SD}=1.08)$, visibility with family/friends scale (mean $=1.5, \mathrm{SD}=0.97)$, visibility with work and public scale (mean $=1.23, \mathrm{SD}=0.98$ ), and sexual orientation conflict scale $($ mean $=1.1, \mathrm{SD}=0.97)$, and the CES-D $($ mean $=19.8, \mathrm{SD}=$ 12.8).

Table 1. Demographic information $(\mathrm{N}=161)$.

\begin{tabular}{|c|c|c|c|c|}
\hline & & Mean (SD) & Frequency & Percentage \\
\hline Age & & $26.7(7.55)$ & & \\
\hline \multirow[t]{3}{*}{ Gender } & Female & & 99 & 61.5 \\
\hline & Male & & 61 & 37.9 \\
\hline & Gender fluid & & 1 & 0.6 \\
\hline \multirow[t]{3}{*}{ Sexual orientation } & Lesbian & & 62 & 38.5 \\
\hline & Gay & & 49 & 30.4 \\
\hline & Bisexual & & 50 & 31.1 \\
\hline \multirow[t]{2}{*}{ Religious } & Yes & & 32 & 19.9 \\
\hline & No & & 129 & 80.1 \\
\hline \multirow[t]{2}{*}{ Education } & Secondary & & 33 & 20.5 \\
\hline & Tertiary or above & & 128 & 79.5 \\
\hline \multirow[t]{5}{*}{ Income } & Below HK\$10000 & & 27 & 16.8 \\
\hline & HK\$10,000 - 20,000 & & 54 & 33.5 \\
\hline & HK $\$ 20,001-30,000$ & & 19 & 11.8 \\
\hline & Above HK\$30,001 & & 17 & 10.6 \\
\hline & Other & & 44 & 27.3 \\
\hline
\end{tabular}


Table 2. Descriptive statistics and correlations between the CES-D and subscales of MOGS.

\begin{tabular}{ccc}
\hline & Mean (SD) & $\begin{array}{c}\text { Pearson correlation } \\
\text { with CES-D }\end{array}$ \\
\hline Family Reaction Subscale & $1.75(1.08)$ & $0.344^{* *}$ \\
Visibility with Family/Friends Subscale & $1.50(0.97)$ & $0.467^{* *}$ \\
Visibility with Work and Public Subscale & $1.23(0.98)$ & $0.441^{* *}$ \\
Sexual Orientation Conflict Subscale & $1.10(0.97)$ & $0.591^{* *}$ \\
$\begin{array}{c}\text { The Center for Epidemiologic Studies } \\
\text { Depression Scale (CES-D) }\end{array}$ & $19.83(12.83)$ & \\
\hline$* * \mathrm{P}<0.001$. &
\end{tabular}

\section{Discussion}

This study aimed to investigate the minority stress and risk of depression among LGB individuals in Chinese societies. The major stressor among the LGB individuals studied was family reaction. In Chinese culture, filial piety is most fundamental of the Confucian values [13]. The traditional Chinese culture on marriage and gender identity affects the perception of LGB individuals on parental attitudes toward marriage [14]. LGB individuals may struggle with the coming out to their parents and perceive this as a stressful event to minimize the impact of the process [15].

Findings are consistent with previous research that minority stressors are related to the psychological distress [16] [17] [18]. Minority stressors were shown to significantly relate with depressive symptoms among the LGB individuals studied. This study indicated that LGB individuals experienced high levels of depressive symptoms. Consistent with previous studies, higher prevalence of emotional distress and depression were reported in sexual minority people [3] [4] [5] [6]. In Chinese societies, prejudice and discrimination against sexual minority people are common [9]. LGB individuals may be victimized and discriminated in hostile social environment and thus leads to poor mental condition [2].

The high levels of depressive symptoms though significantly related to the 4 subscales, the stress level of MOGS ranged from 1.10 to 1.75, which was not too high. One must be noted, the LGB individuals studied were mostly young adults, the high level of depressive symptoms may be also attributed from other stressful events of their daily life, rather than sexual minority related stressors.

Some limitations of the present study must be noted. The use of convenience sample and recruiting participants from social platform of a single organization limited the generalizability of the study results to the general populations of Chinese LGBs. The second limitation concerns the unbalanced gender composition in the sample, as most of the participants $(61.5 \%)$ were female, which was unable to generate differential effect between males and females. 


\section{Conclusion}

Despite the limitations reported, the present study adds knowledge to the literature by documenting the psychological health status of LGB individuals in Asian societies. To date, the majority of research efforts are based on the LGB samples in the Western cultures. There is a dearth of mental health related studies among LGB individuals in Chinese society. The results of this study attempts to serve this purpose. The data revealed that the perceived family reaction in LGBs is associated with their level of depression. The findings suggest the need to focus on culture-specific values in promoting the mental health of LGB individuals within Chinese societies.

\section{Acknowledgements}

The author would like to thank all the participants who participated in the study and the students who helped collect the data. Very special thanks go to the organization, Les concern which has been collaborated in the research.

\section{Conflicts of Interest}

The authors declare no conflicts of interest regarding the publication of this paper.

\section{References}

[1] WHO (2016) FAQ on Health and Sexual Diversity: An Introduction to Key Concepts. World Health Organization, Geneva, 2016.

https://www.who.int/gender-equity-rights/news/20170329-health-and-sexual-divers ity-faq.pdf

[2] Katz-Wise, S.L. and Hyde, J.S. (2012) Victimization Experiences of Lesbian, Gay, and Bisexual Individuals: A Meta-Analysis. The Journal of Sex Research, 49, 142-167. https://doi.org/10.1080/00224499.2011.637247

[3] Zhang, H., Wong, W.C.W., Ip, P., Fan, S. and Yip, P.S.F. (2017) Health Status and Risk Behaviors of Sexual Minorities among Chinese Adolescents: A School-Based Survey. Journal of Homosexuality, 64, 382-396. https://doi.org/10.1080/00918369.2016.1190221

[4] Seil, K.S., Desai, M.M. and Smith, M.V. (2014) Sexual Orientation, Adult Connectedness, Substance Use, and Mental Health Outcomes among Adolescents: Findings from the 2009 New York City Youth Risk Behavior Survey. American Journal of Public Health, 104, 1950-1956. https://doi.org/10.2105/AJPH.2014.302050

[5] Hughes, M. (2017) Health and Well Being of Lesbian, Gay, Bisexual, Transgender and Intersex People Aged 50 Years and over. Australian Health Review, 42, 146-151. https://doi.org/10.1071/AH16200

[6] Barrientos, J., Gómez, F. and Cárdenas, M. (2016) Subjective Wellbeing and Levels of Clinical Symptomatology in a Transwomen Sample and Men Who Have Sex With Men in Chile. Journal of Homosexuality, 63, 1502-1516. https://doi.org/10.1080/00918369.2016.1223346

[7] Meyer, I.H. (2003) Prejudice, Social Stress, and Mental Health in Lesbian, Gay, and Bisexual Populations: Conceptual Issues and Research Evidence. Psychological bulletin, 129, 674-697. https://doi.org/10.1037/0033-2909.129.5.674 
[8] Lee, Y.-T., Yang, H. and Wang, M. (2009) Daoist Harmony as a Chinese Philosophy and Psychology. Peace and Conflict Studies, 16, Article 5.

[9] Chow, P.K.Y. and Cheng, S.T. (2010) Shame, Internalized Heterosexism, Lesbian Identity and Coming out to Others: A Comparative Study of Lesbians in Mainland China and Hong Kong. Journal of Counseling Psychology, 57, 92-104. https://doi.org/10.1037/a0017930

[10] Lewis, R.J. (2001) An Empirical Analysis of Stressors for Gay Men and Lesbian. Journal of Homosexuality, 42, 63-88. https://doi.org/10.1300/J082v42n01_04

[11] Radloff, L.S. (1977) The CES-D Scale: A Self Report Depression Scale for Research in the General Population. Applied Psychological Measurements, 1, 385-401. https://doi.org/10.1177/014662167700100306

[12] Lewinsohn, P.M., Seeley, J.R., Roberts, R.E. and Allen, N.B. (1997) Center for Epidemiological Studies-Depression Scale (CES-D) as a Screening Instrument for Depression among Community-Residing Older Adults. Psychology and Aging, 12, 277-287. https://doi.org/10.1037/0882-7974.12.2.277

[13] Radice, T. (2017) Confucius and Filial Piety. In: Goldin, P.R., Ed., A Concise Companion to Confucius. https://doi.org/10.1002/9781118783863.ch9

[14] Hu, X. and Wang, Y.K. (2013) LGB Identity among Young Chinese: The Influence of Traditional Culture. Journal of Homosexuality, 605, 667-684. https://doi.org/10.1080/00918369.2013.773815

[15] Wang, F.T.Y., Bih, H.-D. and Brennan, D.J. (2009) Have They Really Come out: Gay Men and Their Parents in Taiwan. Culture, Health \& Sexuality, 11, 285-296. https://doi.org/10.1080/13691050802572711

[16] Kelleher, C. (2009) Minority Stress and Health: Implications for Lesbian, Gay, Bisexual, Transgender, and Questioning (LGBTQ) Young People. Counselling Psychology Quarterly, 22, 373-379. https://doi.org/10.1080/09515070903334995

[17] Marshal, M.P., Dietz, L.J., Friedman, M.S., Stall, R., Smith, H.A., McGinley, J., Thoma, B.C., Murray, P.J., D’Augelli, A.R., et al. (2011) Suicidality and Depression Disparities between Sexual Minority and Heterosexual Youth: A Meta-Analytic Review. The Journal of Adolescent Health: Official Publication of the Society for Adolescent Medicine, 49, 115-123. https://doi.org/10.1016/j.jadohealth.2011.02.005

[18] Meyer, I. (1995) Minority Stress and Mental Health in Gay Men. Journal of Health and Social Behavior, 36, 38-56. http://www.jstor.org/stable/2137286 https://doi.org/10.2307/2137286 